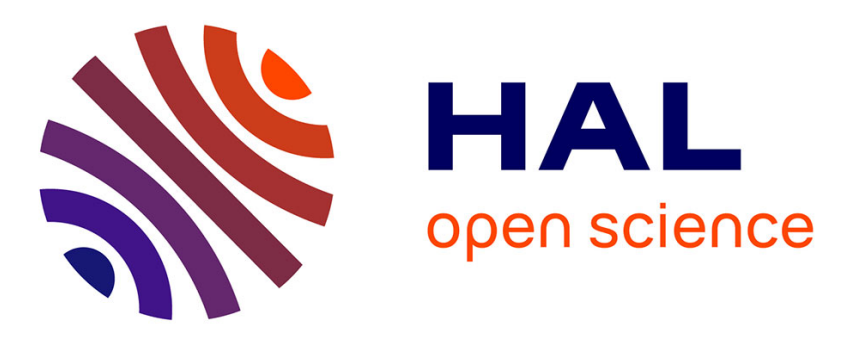

\title{
A survey of V2V channel modeling for VANET simulations
}

\author{
Hervé Boeglen, Benoit Hilt, Pascal Lorenz, Jonathan Ledy, Anne-Marie \\ Poussard, Rodolphe Vauzelle
}

\section{- To cite this version:}

Hervé Boeglen, Benoit Hilt, Pascal Lorenz, Jonathan Ledy, Anne-Marie Poussard, et al.. A survey of V2V channel modeling for VANET simulations. The 8th International Conference on Wireless Ondemand Network Systems and Services - WONS 2011, Jan 2011, Bardonecchia, Italy. hal-00658989

\section{HAL Id: hal-00658989 \\ https://hal.science/hal-00658989}

Submitted on 13 Jan 2012

HAL is a multi-disciplinary open access archive for the deposit and dissemination of scientific research documents, whether they are published or not. The documents may come from teaching and research institutions in France or abroad, or from public or private research centers.

$$
\text { Copyright }
$$

L'archive ouverte pluridisciplinaire HAL, est destinée au dépôt et à la diffusion de documents scientifiques de niveau recherche, publiés ou non, émanant des établissements d'enseignement et de recherche français ou étrangers, des laboratoires publics ou privés. 


\section{A survey of V2V channel modeling for VANET simulations}

\author{
Hervé Boeglen, Benoît Hilt, Pascal Lorenz \\ Laboratoire MIPS-GRTC \\ EA2332 \\ Université de Haute Alsace, France \\ [herve.boeglen, benoit.hilt, pascal.lorenz]@uha.fr
}

\author{
Jonathan Ledy, Anne-Marie Poussard, \\ Rodolphe Vauzelle \\ Laboratoire XLIM-SIC \\ UMR CNRS 6172 \\ Université de Poitiers, France \\ [ledy,poussard,vauzelle]@sic.univ-poitiers.fr
}

\begin{abstract}
Most Vehicle to Vehicle (V2V) network protocols are evaluated by simulation. However in most network simulators, the physical layer suffers from a lack of realism. Therefore, realistic $\mathrm{V} 2 \mathrm{~V}$ channel modeling has become a crucial issue in Intelligent Transportation Systems (ITS) networks. V2V channels are known to exhibit specific features which imply the design of new simulation models. In this survey paper, we first recall the main physical features of such wireless time and frequency dispersive channels. Next, three "simulation-ready" V2V channel models found in the literature are reviewed. Finally, two complete VANET simulation frameworks are presented. They illustrate the importance of a realistic channel and physical layer modeling in vehicular networking.
\end{abstract}

Keywords: wireless V2V channels, modeling, characterization, simulation, VANET.

\section{INTRODUCTION}

In recent years, Vehicle-to-Vehicle (V2V) wireless communications have received a lot of attention as they are going to be a crucial issue in Intelligent Transportation Systems (ITS). In particular, different applications will emerge enabled by the exchange of information between cars. The main ones concern the enhancement of road safety and the reduction of the traffic impact on the environment. In the near future, this technology will also allow the setting of car networks which are going to exchange high rate multimedia information for entertainment applications. These networks are called Vehicular Ad-Hoc Networks (VANETs). In order to transmit information reliably on rapidly changing vehicular channels one has to rely on a robust physical layer. This is precisely the challenge the $802.11 \mathrm{p}$ working group is facing in designing a physical layer standard for $\mathrm{V} 2 \mathrm{~V}$ communications [1]. This physical layer has to be evaluated by means of realworld measurements but also by means of less costly simulations implementing realistic channel models.

Finding an accurate channel model for VANETs is still a research issue. Indeed, it has been shown in several papers that vehicular wireless channels exhibit specific characteristics that makes them quite different from the very well characterized mobile telephony channels [2][3]. Therefore, the aim of this survey paper is firstly to present the characteristics of wireless $\mathrm{V} 2 \mathrm{~V}$ channels which are going to influence the digital physical layer. To achieve this goal we will first give a quick summary of the most important parameters of wireless time and frequency dispersive channels. In the second part of this paper, we review three different "ready to use" channel models designed for $\mathrm{V} 2 \mathrm{~V}$ communications found in the literature. We conclude this second part with the presentation of two complete VANET simulation frameworks which allow simulating complex vehicular mobility scenarios. At the end of this paper we expect that the reader will have enough knowledge in this field to be able to choose the right channel model/simulator for the $\mathrm{V} 2 \mathrm{~V}$ situation he wants to simulate.

\section{WIRELESS CHANNEL MODELING AND CHARACTERIZATION BACKGROUND}

The modeling of wireless channels has several decades of history behind it. For reasons of space and brevity we will only give an overview of the subject in this section. For a more detailed description of this field the reader is referred to [4] [5] and [6]. It is well known that channel models fall into two classes: deterministic and stochastic. Deterministic channel modeling methods allow determination of the fieldstrength at all points and times in an environment by solving Maxwell's equations. These methods fall into two categories. The accurate methods solve Maxwell's equations in some sort of discretized way (e.g. the Method of Moments). The asymptotic methods use approximations to Maxwell's equations (e.g. ray-launching and ray-tracing methods) [6]. The results obtained are very realistic but are site specific. Moreover, deterministic channel simulators require high computational times. On the other hand, statistical channel models are computationally efficient and do not try to mimic a precise situation but rather attempt to faithfully emulate the variations of the main channel effects.

In a wireless system, the transmitted signals can propagate to the receiver via different paths. This effect is called multipath propagation. It comes from the fact that the transmitted waves interact with the objects present in the environment (e.g. buildings, trees etc.) which causes 
reflections, diffractions and scattering. This effect is commonly observed on the Channel Impulse Response (CIR) $h(\tau)$ of the channel at the receiver side. An example of a CIR made up of 6 paths (or taps) is given in Figure 1.a.
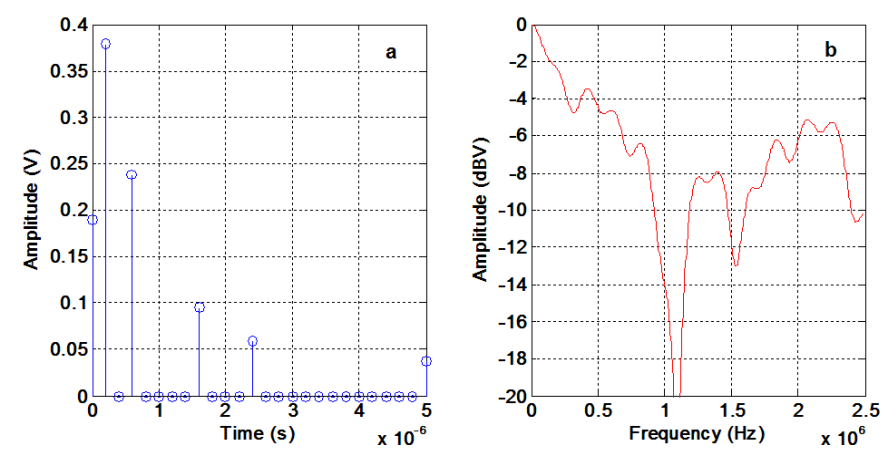

Figure 1. Example of a CIR in the delay (a) and frequency (b) domains

One can notice that the different paths do not arrive at the same time $\tau$ at the receiver (because there travel paths have different length) and that they do not have the same amplitude (and also not the same phase, which is not represented in Figure 1.a). This could include a direct or Line-Of-Sight (LOS) path. Please note that this representation of the CIR is a discretized version of the continuous physical CIR. This remark leads to the important notion of resolvable path. Indeed, a digital receiver having a sampling time Ts, cannot distinguish between echoes arriving at $\tau$ and $\tau+\Delta \tau$ if $\Delta \tau<<$ Ts. This condition can lead to a one path CIR. In that case, the system is considered as narrowband and the channel is non-frequency selective. On the other hand, if Ts is high enough to allow distinguishing a multiple path CIR, we have a wideband system and the channel can be considered as frequency selective. Please keep in mind that since Ts is always finite, it is physically impossible for a practical system to discriminate all the subpaths composing a CIR.

Multipath propagation and delay dispersion (if the CIR is made up of more than one path) causes frequency selectivity as can be observed in Figure 1.b which is obtained by Fourier Transformation (FT) of the CIR. From a signal processing point of view, this means that if the transmitted signal has a large bandwidth its spectrum is going to be distorted by the channel. To characterize the frequency selectivity of a CIR one finds generally the maximum delay spread $\tau_{\max }$ which is the delay of the last path in the CIR or, in the case of a statistical characterization, the Root Mean Square (RMS) value of the delay spread $\tau_{\text {RMS }}[6] . \tau_{\text {RMS }}$ is related to the coherence bandwidth $\mathrm{Bc}$ by relation (1) shown below. Bc represents the frequency band on which the channel can be considered as non-selective.

$$
\mathrm{Bc} \approx 0.2 / \tau_{\mathrm{RMS}}
$$

Up to now we have considered that there was no mobility i.e. that the CIR was not time dependant. But when the receiver and/or the transmitter or the interacting objects in the environment move, it is easy to understand that the parameters of the CIR change with time (delays, amplitudes and phases). In particular the change in amplitude can be very important yielding severe fading of the signal. This situation is represented in Figure 2. As can be observed, some components can even disappear from the CIR showing a path obstruction situation.

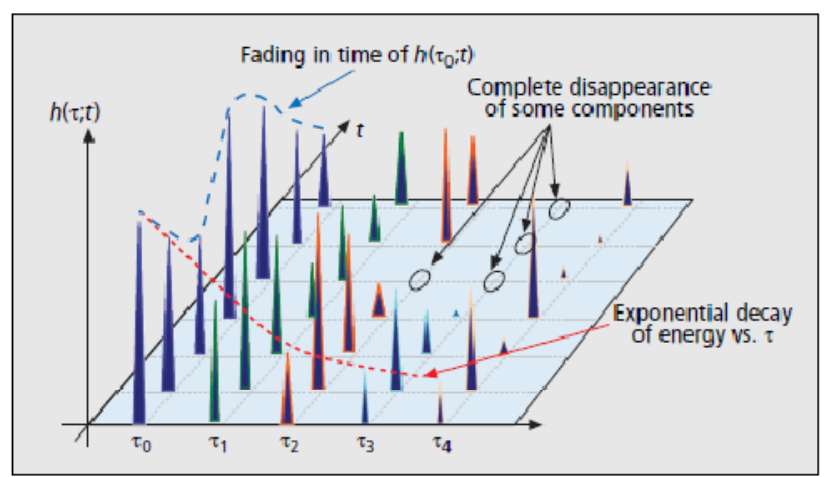

Figure 2. Illustration of the time variance of the CIR (reproduced from [2]).

An important function which characterizes this time variation in the Doppler frequency domain is the scattering function $\mathrm{S}(\tau, v)$ obtained by FT of the CIR on the $t$ variable. Figure 3 shows an example of a scattering function for one path obtained in a $\mathrm{V} 2 \mathrm{~V}$ situation.

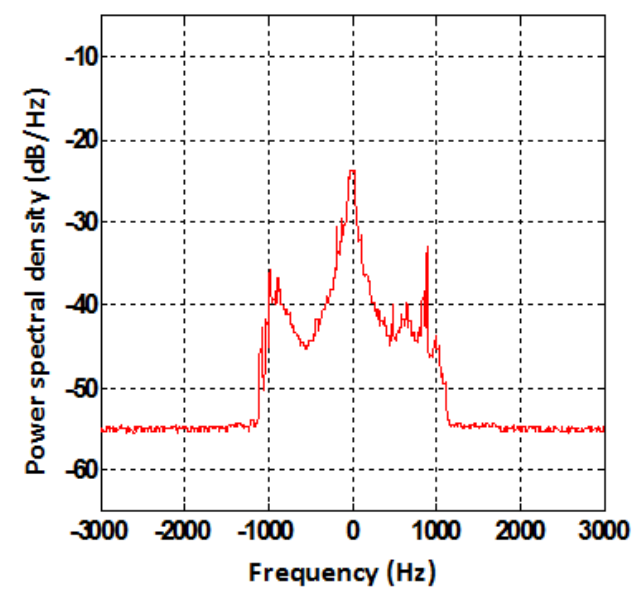

Figure 3. An example of a measured $\mathrm{V} 2 \mathrm{~V}$ scattering function $\mathrm{S}(\tau, v)$

The width of this function in the Doppler variable is called the Doppler spread $\mathrm{f}_{\mathrm{Dmax}}$ and measures the amount by which the channel spreads a transmitted tone in frequency. $f_{D \max }$ is related to the mobile speed by:

$$
\mathrm{f}_{\mathrm{Dmax}}=(\mathrm{v} / \mathrm{c}) \cdot \mathrm{f}_{\mathrm{c}}
$$

where $\mathrm{v}$ is the speed of the mobile, $\mathrm{c}$ the speed of light and fc the carrier frequency. The fact that $S(\tau, v)$ depends on $\tau$ implies that each path of the CIR has an associated scattering function $\mathrm{S}(\tau, v)$ which can vary in shape and Doppler spread. The time selectivity of the channel is often related to the coherence time $T_{C}$ which is linked to the Doppler spread by equation (3). $T_{C}$ 
represents the amount of time during which the channel parameters can be considered as stable.

$$
\mathrm{T}_{\mathrm{C}} \approx 1 / \mathrm{f}_{\mathrm{Dmax}}
$$

We will finish this rapid overview of the main physical effects observed on a mobile wireless channel by underlining two important theoretical frameworks in which they fall. Firstly, one can rely on the linear time variant system theory to characterize the channel: a channel can indeed be seen as a linear filter [7]. Secondly, a wireless channel is a stochastic process which implies that it has to be characterized by autocorrelation and Power Spectral Density (PSD) functions. These functions are related by Fourier Transformations [7]. Most practical models assume that the stochastic channel is Wide Sense Stationary (WSS) meaning that its statistical properties do not change over time. Moreover they also assume that the taps of the CIR are uncorrelated: this is known as the Uncorrelated Scattering (US) assumption [7]. We will see in what follows that in many VANET situations the WSSUS assumption does not hold.

When it comes to implementation of wideband statistical channel models, the most common simulation structure used is the Tapped Delay Line (TDL) filter as shown in Figure 4. It is a linear Finite Impulse Response (FIR) filter model where the filter coefficients or tap weights are stochastic processes.

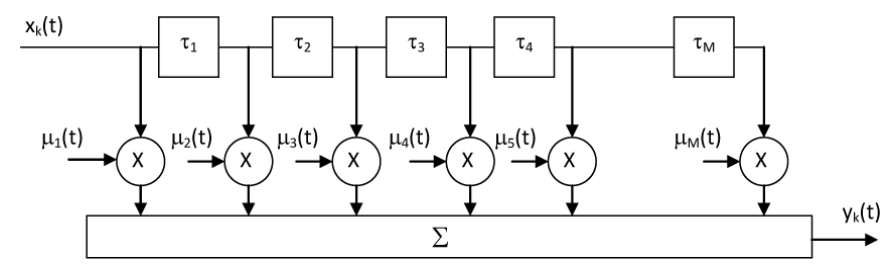

Figure 4. The TDL structure

The input data (i.e. the digital modulation samples) is denoted $x_{k}(t)$ and $y_{k}(t)$ represents the output of the channel. The blocks labeled $\tau_{\mathrm{k}}$ denote the delays and in digital systems they are typically equal to the symbol period. The tap weights $\mu_{k}(t)$ are complex numbers of the form $\mu_{k}(t)=\alpha_{k}(t) e^{j \Phi k(t)}$. The tap amplitude $\alpha_{k}(t)$ is generated according to a statistical distribution that fits the measured amplitude. The most common one is the Rayleigh distribution. Other tap amplitude distributions include the Ricean, Nakagami and Weibull distributions [16]. Unless there is a dominant LOS component, the tap phases $\Phi_{\mathrm{k}}(\mathrm{t})$ are modeled as uniformly distributed over $[0,2 \pi]$. In order to simulate the Doppler effect, the tap weights samples $\mu_{\mathrm{k}}(\mathrm{t})$ are finally passed into a filter whose cutoff frequency corresponds to $\mathrm{f}_{\mathrm{Dmax}}$ and whose shape is similar to the measured scattering function $S(\tau, v)$ [8]. This filtering can either be performed in the time or in the frequency domain [8].

In conclusion, in order to simulate a wireless channel with the TDL structure one needs to know the number $M$ of taps in the CIR, their respective delays $\tau_{\mathrm{k}}$, the statistical distribution of the amplitude for each tap, the shape and Doppler frequency limit of the scattering function $S(\tau, v)$ for each tap.

Please note that TDL modeling only takes into account the so called small scale effects of the channel which are observed on moving distances on the order of the carrier frequency wavelength $\lambda$. For medium scale effects (moving distances between 10 to $40 \lambda$, i.e. shadowing effects) or large scale effects (propagation loss) one has to simulate these features separately with appropriate models [6].

We have now all the ingredients needed to fully understand the three channel models specifically designed for VANETs that we are going to evaluate in the next section.

\section{V2V CHANNEL MODELS}

As we are going to see in what follows, V2V channel simulations still involve the TDL structure. However, the TDL parameters have to be adapted to $\mathrm{V} 2 \mathrm{~V}$ channel conditions. These conditions differ from the ones found for mobile cellular communication channels. The authors of reference [3] underline the following main differences:

- In V2V communications the transmitter (TX) and the receiver $(\mathrm{RX})$ are at the same height. This implies that the main propagation mechanisms of the multipath components are different. The consequences are seen on the Angles of Arrival (AoA) and Angles of Departures (AoD) of the travelling waves which implicates scattering function shapes quite different from what is observed in mobile telephony.

- In a V2V channel, both the TX and the RX as well as many scatterers in the environment are moving. This implicates faster channel fluctuations and that commonly used assumptions on stationarity (WSSUS) are usually not valid.

- $\mathrm{V} 2 \mathrm{~V}$ communications operate mostly in the $5 \mathrm{GHz}$ band whereas cellular communications occur between 700-2100 MHz. The consequence is a higher signal attenuation while specific propagation processes are less efficient (e.g. diffraction).

- Statistical channel modeling is related to a propagation environment. The following V2V specific environments are generally found in the literature [3]:

- Highways having 2 to 6 lanes with speed ranging from 25 to more than $40 \mathrm{~m} / \mathrm{s}$ and different traffic densities.

- Rural streets with two lanes and speeds up to 30 $\mathrm{m} / \mathrm{s}$. There are few buildings and density of vehicles is low.

- Suburban streets with one or two lanes. The traffic density is generally low and speed is limited to about $15 \mathrm{~m} / \mathrm{s}$ or less.

- Urban streets. There are characterized by wider streets and higher traffic density than suburban streets. 
When it comes to simulate V2V channels one has got two choices. The first possibility is to rely on a deterministic channel simulator which presents the advantage of taking into account a great number of situations in realistically modeled scenes though at the expense of a higher computational time. The other possibility is to select a suitable stochastic model in the literature. Such a model is generally derived from intensive measurements. At the time of writing, we found three major implementations that we are going to describe in what follows. We have chosen these particular three because they are "simulation-ready" since their authors provide very clear implementation recipes.

Whatever the V2V channel simulator chosen, it has to be integrated in a simulation platform which will allow evaluating real-world situations. We will present two such implementations in section IV.

\section{A. The channel models used by the $802.11 p$ working group}

These are traditional TDL WSSUS implementations based on the work of Acosta-Marum et al. [9]. They performed intensive measurements in different $\mathrm{V} 2 \mathrm{~V}$ situations and derived a set of 6 very simple channel models suitable for channel emulators [10]. In reference [9] the authors provide in a table all the parameters needed to simulate these channels by using the TDL structure. V2V complex Doppler shapes are obtained by superposing elementary shapes (Flat, Round, Classic $3 \mathrm{~dB}$ and Classic 6dB). A very good and very well documented implementation based on the IT ++ library [11] can be found on the Internet [12]. Although these channel models have been adopted by the $802.11 \mathrm{p}$ working group, they suffer from important shortcomings. Indeed, these channel models do not take into account two main characteristics of V2V channels, i.e. the non-stationarity of the CIR and the correlation between CIR taps. Several authors report that the WSSUS assumption in $\mathrm{V} 2 \mathrm{~V}$ situations leads to erroneous results [3][13]. It is still not very clear whether non-stationary conditions will be worse or better than WSSUS conditions.

\section{B. The Sen-Matolak implementation}

This channel model is described in great details in reference [13]. The authors conducted V2V measurements in five cities of the Ohio state in the USA in several traffic conditions. From these measurements they derived non stationary correlated scattering TDL channel models. To account for the non-stationarity, they model the appearance/disappearance of the CIR taps by a persistence process which is implemented by a two-state Markov process. This process is specified by two matrices: the transition (TS) matrix and the steady-state matrix (SS). These matrices are defined as follows:

$$
T S=\left[\begin{array}{ll}
P_{00} & P_{01} \\
P_{10} & P_{11}
\end{array}\right] \quad S S=\left[\begin{array}{l}
P_{0} \\
P_{1}
\end{array}\right]
$$

Each element $\mathrm{P}_{\mathrm{ij}}$ in matrix TS gives the probability of going from state $i$ to state $j$, and each $S S$ element $P_{j}$ gives the "steady-state" probability of the jth state. An example of such a persistence process for taps 5 and 8 from an urban CIR is shown in Figure 5.
In this channel model, the correlation between taps is also taken into account and the authors provide the values obtained from the measurements in their paper. They have also found that the amplitude distribution of the taps was best described by a Weibull distribution [15].
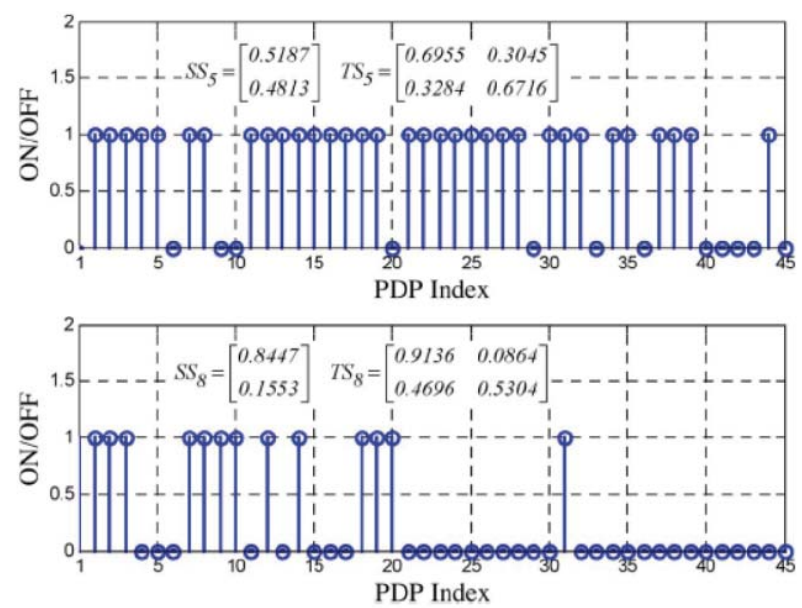

Figure 5. Sample persistence processes for two taps (reproduced from [13])

When the $\beta$ parameter of this distribution is smaller than 2 , which was often the case in the measurements, the fading is more severe than the one obtained with a Rayleigh distribution. The authors compare their models to a traditional WSSUS model which, because of the uncorrelated scattering, is shown to be more frequency selective than the non-WSSUS one. One shortcoming of this model is the simplistic implementation of the Doppler effect: a simple lowpass filtering is performed on the Weibull generated samples. Finally, the authors provide all the needed parameters to implement their models and give in a separate paper an algorithm that can generate multivariate Weibull distributions with arbitrary fading and correlation parameters in order to implement their channel models [17].

\section{A Geometry-based Stochastic MIMO Channel Model (GSCM) for V2V communications [18]}

This kind of channel modeling consists in placing scatterers (i.e. interacting objects) at random, according to certain statistical distributions and assigning them scattering properties. Then, the signal contributions of the scatterers are determined from a simplified ray-tracing, and finally the total signal is summed up at the receiver side. Please note that the final implementation consists again in a TDL model which is fed by the parameters obtained from the GSCM settings. This type of channel model is a double-directional channel model [19] and is natively MIMO since it takes into account the arrival and departure angles of the propagated waves. It can also handle non-WSSUS conditions and provides delay and Doppler spectra. This GSCM model for V2V is an evolution of the model initially described by Molisch and co-authors in [20] and [21]. The authors of this paper have performed a measurement campaign which has allowed them to identify key parameters of the V2V channel. The V2V CIR can indeed 
be divided into four parts: (i) the LOS component, (ii) discrete components stemming from reflections off mobile scatterers (i.e. passing cars and lorries), (iii) discrete components stemming from reflections off static scatterers (e.g. road signs) and (iv) diffuse components (produced by the environment along the roads). It is also noted that in many cases the WSSUS assumption is violated. This is typically observed when discrete components move through many delay taps during a measurement (i.e. the taps are correlated). Last but not least, it has also been observed that the Doppler spectrum undergoes significant changes during a measurement. This is in contradiction with the fixed Doppler spectrum implementations of Acosta-Marum et al. [9] and Sen et al [13]. The GSCM V2V model has another significant advantage over the two previous implementations since it is able to simulate MIMO antenna systems. Indeed, this technology has been shown to be very robust in severe fading situations. Moreover, the use of several antennas will allow to take advantage of the so-called diffuse components which have been shown to carry important energy [22]. For implementation issues of this model we refer the reader to references [20] and [18].

We have just reviewed three realistic "simulation-ready" $\mathrm{V} 2 \mathrm{~V}$ channel models. In order to evaluate them in VANET situations, a $802.11 \mathrm{p}$ compliant physical layer has also to be implemented. Finally, this combination (channel model + physical layer) when linked to a network simulator allows for the simulation of realistic VANET scenarios. We are going to present two such VANET simulation platforms in the next section.

\section{TOWARDS A REALISTIC VANET SIMULATOR}

In reference [23], we propose a semi-deterministic channel model called UM-CRT which uses a deterministic propagation simulator called Communication Ray Tracer (CRT) together with the Spatial Channel Model Extended (SCME) statistical channel model [24]. The SCME describes three different environments. The Urban Microcell (UM) scenario has been selected because it considers communication distances of less than one kilometer which is common in VANET situations.

The CIRs obtained by CRT are used to parameterize the SCME-UM statistical channel model. Because UM-CRT is born from the merging of two other models, the best way to describe it is to explain thoroughly its simulation procedure. Figure 6 depicts both CRT and SCME-UM, respectively in the left and right parts with solid arrows. UM-CRT is depicted with dashed arrows.

When using UM-CRT, we determine if we are in a LOS or NLOS situation from the CIR obtained with CRT (see dashed arrow on the upper center of Figure 6). The LOS-NLOS criterion is used as a first approach. We are currently working on the possibility to use more relevant criteria to better characterize the VANET propagation channel. Using the criterion mentioned before, together with a corresponding transmitter-receiver distance, we choose an equivalent SCMEUM CIR which has been pre-calculated.

The simulation works in two steps. The first step consists in a pre-processing stage which still requires a high computation time mainly due to the use of the CRT deterministic channel simulator. However, the second step is very fast since it only consists of reading the CRT CIR to determine the LOS-NLOS criterion, then reading the corresponding SCME-UM CIR coefficients according to the distance between the transmitter and the receiver.

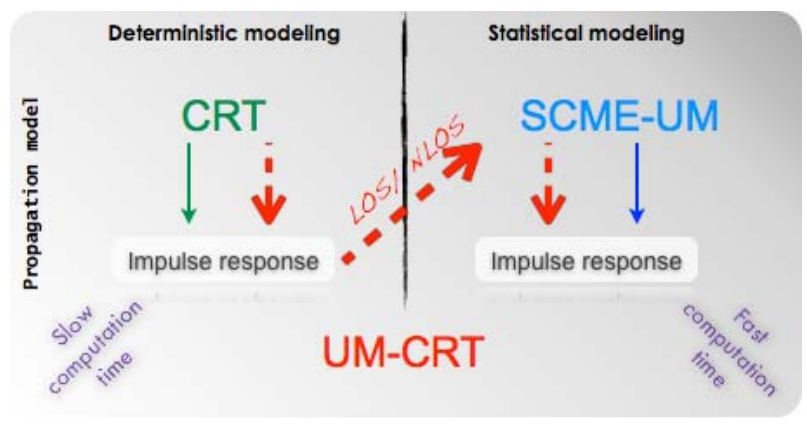

Figure 6. The UM-CRT model.

In order to evaluate this model in VANET situations, it has been integrated in the network simulator NS-2 [14]. Fully compliant $802.11 \mathrm{p}$ and $802.11 \mathrm{n}$ physical layers using the IT++ library have also been implemented. We have performed simulations in SISO and MIMO modes in the $5 \mathrm{Ghz}$ band. In order to have some typical cases for comparing UM-CRT to CRT, four representative urban mobility scenarios have been generated by VanetMobiSim [25]. Figure 7 shows the results in terms of Packet Delivery Ratio (PER) obtained for one scenario in the SISO and the MIMO cases. One can observe that the semi-deterministic model (UM-CRT) comes very close to the deterministic channel simulator (CRT). As expected, one can also notice the significant improvement brought by the $2 \times 2$ MIMO configuration (Alamouti scheme).

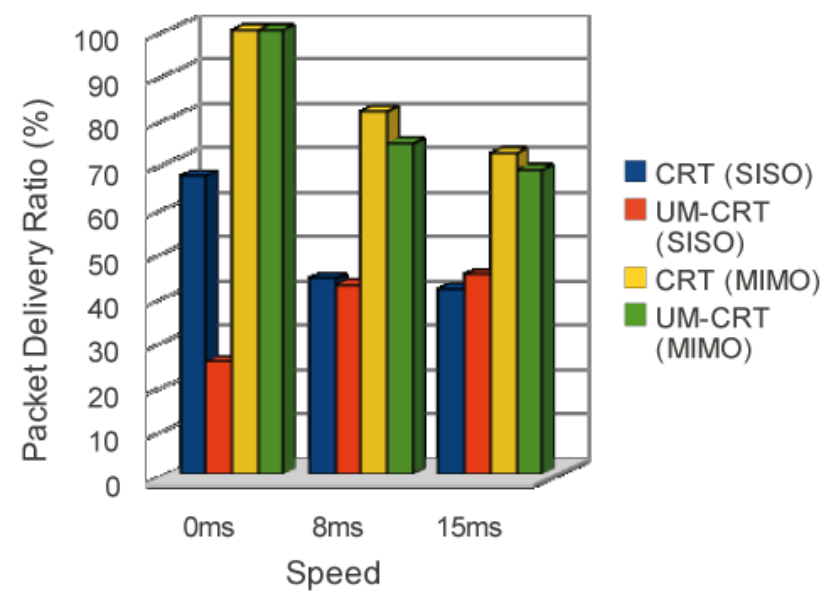

Figure 7. Evaluation of the UM-CRT model in SISO and MIMO configurations.

We started this work on designing a realistic VANET simulator because we noticed that many simulations reported in the wireless networking literature contained simplistic abstraction of the physical layer which lead to erroneous results. This work is therefore an attempt to "bridge the gap between physical layer emulation and network simulation" [26]. 
The authors of reference [26] came to the same conclusion as us and designed PhySim-WiFi. This implementation is a very realistic 802.11 ( $\mathrm{a}, \mathrm{g}$ and $\mathrm{p}$ ) physical layer for the network simulator NS-3 going as low as the OFDM synchronization and channel estimation algorithms usually implemented in wireless chipsets. Physim-WiFi has been tested against real 802.11 OFDM-based communication chipsets by using the CMU network emulator [27]. Figure 8 shows the results in terms of Frame Reception Ratio as a function of the Signal to Interference-Noise Ratio (SINR). The PhySim-WiFi implementation is compared to the plain vanilla NS-3 and CMU emulator at 6 and 24Mbps.

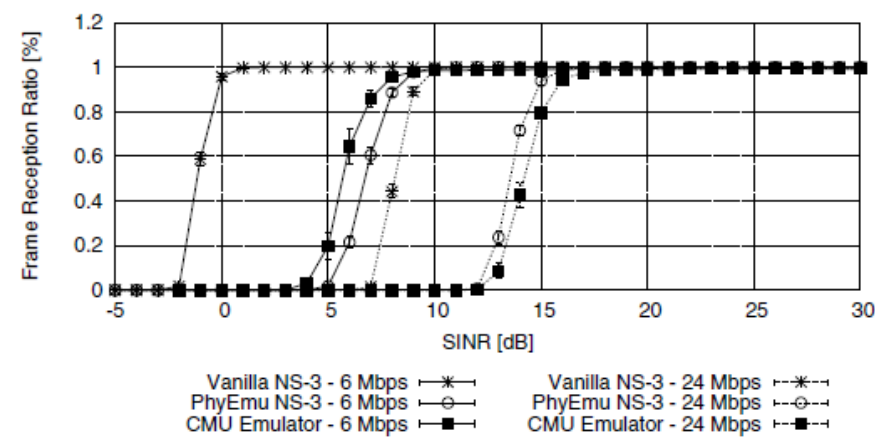

Figure 8. Evaluation of the PhySim-Wifi realistic 802.11p physical layer integrated in NS-3[26].

Please note the huge difference ( $7 \mathrm{~dB}$ in SINR!) between the plain vanilla NS-3 implementation and the realistic ones. This really demonstrates the key role of the physical layer in wireless networking! This realistic physical layer implementation together with the channel models described in section III.A is publicly available on the Internet [27].

From the previous discussion one can now easily understand that accurate $\mathrm{V} 2 \mathrm{~V}$ channel modeling together with a realistic physical layer implementation is essential in the simulation of VANET scenarios. This issue is also of great importance if one intends to implement cross-layer optimisation strategies.

\section{CONCLUSION}

In this paper we have first presented the main physical effects occurring on time and frequency selective wireless channels and how they are taken into account in the wideband TDL filter structure. Then we have underlined the V2V channels specificities and particularly the fact that they are usually non-stationary and reviewed three different "simulation-ready" V2V channel models. Next, we presented two complete VANET simulation environments and concluded on the strong impact of the $\mathrm{V} 2 \mathrm{~V}$ propagation channel on VANET real-time communications although several network simulations works still underestimate its importance.

However, many open topics remain. The small amount of available $\mathrm{V} 2 \mathrm{~V}$ measurements does not allow the formulation of statistically significant statements about real-world V2V channels. Moreover, VANET environments characteristics vary regionally. Measurements have therefore to be performed in different parts of the world.

In order to increase the robustness of $\mathrm{V} 2 \mathrm{~V}$ communications, multi-antenna systems have also to be considered. This issue implies to measure and design channel models that take into account the angle domain (like the GSCM channel implementation discussed earlier). Many people of this domain think that MIMO is going to be a natural evolution of the $802.11 \mathrm{p}$ standard.

The $802.11 \mathrm{p}$ physical layer, which was adapted from the 802.11a standard, initially designed for indoor, slowly varying conditions, is far from being optimum in V2V situations. A known example concerns the 802.11 p packet length which in some V2V situations can exceed the channel coherence time $\mathrm{T}_{\mathrm{C}}$ [23]. These situations underline the need for the study and design of more efficient digital communication strategies and in particular in the field of channel estimation algorithms [28].

\section{REFERENCES}

[1] "IEEE Draft Standard for Information Technology Telecommunications and information exchange between systems - Local and metropolitan area networks - Specific requirements - Part 11: Wireless LAN Medium Access Control (MAC) and Physical Layer (PHY) specifications Amendment : Wireless Access in Vehicular Environments", IEEE Unapproved Draft Std P802.11p/D11.0, Mar. 2010.

[2] D. W. Matolak, "Channel Modeling for Vehicle-To-Vehicle Communications", IEEE Communications Magazine, vol.46, no.5, pp.76-83, May 2008.

[3] A. Molisch, F. Tufvesson, J. Karedal, C. Mecklenbrauker, "A survey on vehicle-to-vehicle propagation channels", IEEE Wireless Communications, vol.16, no.6, pp.12-22, Dec. 2009.

[4] J. D. Parsons, "The mobile radio propagation channel", $2^{\text {nd }}$ edition, Wiley, 2000.

[5] M. Paetzold, "Mobile fading channels", Wiley, 2002.

[6] A. Molisch, "Wireless communications", Wiley-IEEE Press, 2005.

[7] P. Bello, "Characterization of Randomly Time-Variant Linear Channels", IEEE Transactions on Communications Systems, vol.11, no.4, pp.360-393, Dec. 1963.

[8] M.C. Jeruchim, P. Balaban, K.S.Shanmugan, "Simulation of Communication Systems", $2^{\text {nd }}$ edition, Springer, 2000.

[9] G. Acosta-Marum, M. A. Ingram, "Six Time- and FrequencySelective Empirical Channel Models for Vehicular Wireless LANs", IEEE 66th Vehicular Technology Conference, 2007, Fall. 2007, vol., no., pp.2134-2138, 30 Sept.- 3 Oct. 2007.

[10] http://www.spirent.com/Solutions-Directory/SR5500.aspx

[11] http://itpp.sourceforge.net/current/

[12] http://sourceforge.net/apps/mediawiki/physlayersim/index.php? $\underline{\text { title}=\text { Main Page }}$

[13] I. Sen, D. W. Matolak, "Vehicle-Vehicle Channel Models for the 5-GHz Band", IEEE Transactions on Intelligent Transportation Systems, volume 9, issue 2, June 2008.

[14] http://www.isi.edu/nsnam/ns/ 
[15] M. K. Simon, M. Alouini, "Digital communication over fading channels", Wiley, 2005.

[16] M. K. Simon, "Probability Distributions Involving Gaussian Random Variables", Springer, 2006.

[17] D. W. Matolak, I. Sen, W. Xiong, "Generation of multivariate Weibull random variates" Communications, IET, vol.2, no.4, pp.523-527, April 2008.

[18] J. Karedal, F. Tufvesson, N. Czink, A. Paier, C. Dumard, T. Zemen, C. F. Mecklenbrauker and A. F. Molisch, "A geometrybased stochastic MIMO model for vehicle-to-vehicle communications", IEEE Transactions on Wireless Communications, volume 8, n7, pp.3646-3657, July 2009.

[19] M. Steinbauer, A. F. Molisch, E. Bonek, "The doubledirectional radio channel", Antennas and Propagation Magazine, IEEE, vol.43, no.4, pp.51-63, Aug 2001.

[20] A. F. Molisch, A Kuchar, J. Laurila, K. Hugl, R. Schmalenberger, "Geometry-based directional model for mobile radio channels-principles and implementation", European Transactions on Telecommunications, Vol. 14, Issue 4, pages 351-359, July/August 2003.

[21] A. F. Molisch, "A generic model for MIMO wireless propagation channels in macro- and microcells", IEEE Transactions on Signal Processing, vol.52, no.1, pp. 61- 71, Jan. 2004.

[22] Y. Zhou, X. Yin, N. Czink, T. Zemen, F. Liu, "Evaluation of doppler-delay properties of diffuse components in vehicular propagation channels", 2nd IEEE Conference on Wireless Access in Vehicular Environments (WAVE2009), Tongji University, Shanghai, China, Dec. 21-22, 2009.

[23] J. Ledy, H. Boeglen, A. Poussard, B. Hilt, R. Vauzelle, "A semi-deterministic channel model for VANETs simulations", The 10th International on ITS Telecommunication, ITST 2010, Kyoto, Japan, 9-10 November 2010.

[24] 3GPP, "Spatial channel model for MIMO simulations", TR 25.996 V9.0.0 (2009-12) [Online]. Available: http://www.3gpp.org/ftp/Specs/archive/25 series/25.996/25996 -900.zip.

[25] http://vanet.eurecom.fr/

[26] S. Papanastasiou, J. Mittag, E. Strom, H.Hartenstein, "Bridging the Gap between Physical Layer Emulation and Network Simulation," Wireless Communications and Networking Conference (WCNC), 2010 IEEE , vol., no., pp.1-6, 18-21 April 2010.

[27] http://dsn.tm.uni-karlsruhe.de/english/ns3-physim.php

[28] L. Bernadó, N. Czink, T. Zemen, P. Belanović, "Physical Layer Simulation Results for IEEE 802.11p Using Vehicular NonStationary Channel Model", Communications Workshops (ICC), 2010 IEEE International Conference on Communication, pp.1-5, 23-27, May 2010. 